PROCEEDINGS OF THE

AMERICAN MATHEMATICAL SOCIETY

Volume 134, Number 10, October 2006, Pages 2905-2911

S $0002-9939(06) 08318-3$

Article electronically published on April 11, 2006

\title{
ENTROPY FOR AUTOMORPHISMS OF FREE GROUPS
}

\author{
MARIE CHODA
}

(Communicated by David R. Larson)

\begin{abstract}
Let $\sigma$ be the automorphism of the free group $F_{\infty}$ which is arising from a permutation of the free generators of $F_{\infty}$. The $\sigma$ naturally induces the automorphism $\hat{\sigma}$ of the reduced $C^{*}$-algebra $C_{r}^{*}\left(F_{\infty}\right)$, and also the automorphism $\overline{\hat{\sigma}}$ of the group factor $L\left(F_{\infty}\right)$. We show that the Brown-Germain entropy $h a(\sigma)$ is zero. This implies that the Brown-Voiculescu topological entropy $h t(\hat{\sigma})$, the Connes-Narnhofer-Thirring dynamical entropy $h_{\phi}(\hat{\sigma})$ and the Connes-Størmer entropy $H(\overline{\hat{\sigma}})$ are all zero.
\end{abstract}

\section{INTRODUCTION}

Two notions of entropy for automorphisms were extended to non-commutative framework in the theory of operator algebras from the ergodic theory.

The dynamical entropy $H(\alpha)$ was introduced by Connes and Størmer in [6] as an extended version of the Kolmogorov and Sinai invariant for an automorphism $\alpha$ of a finite von Neumann algebra $M$. The value depends on a given normal faithful tracial state $\tau$ of $M$ which is preserved by the automorphism $\alpha$.

Replacing the trace $\tau$ to an $\alpha$-invariant state $\phi$, Connes-Narnhofer-Thirring defined in [7] the entropy $h_{\phi}(\alpha)$ for an automorphism $\alpha$ of a unital $C^{*}$-algebra $A$ as an extension of $H(\alpha)$. They proved that if $M$ is the von Neumann algebra generated by the image of $A$ under the GNS representation of $\phi$, and if $\bar{\alpha}$ and $\bar{\phi}$ are the canonical extensions of $\alpha$ and $\phi$, then $h_{\phi}(\alpha)=h_{\bar{\phi}}(\bar{\alpha})$. Moreover if $\phi$ is a tracial state, then $h_{\bar{\phi}}(\bar{\alpha})=H(\bar{\alpha})$.

The topological entropy $h t(\alpha)$ is defined for an automorphism $\alpha$ of unital $C^{*}$ algebras which have the approximation property. The definition is based on the approximation property. It was invented by Voiculescu 15 for nuclear $C^{*}$-algebras, and extended by Brown 2 to exact $C^{*}$-algebras.

These two kinds of "entropy" always satisfy that $h_{\phi}(\alpha) \leq h t(\alpha)$ by Dykema $[8$.

Discrete groups are one of the most basic objects producing many very interesting examples of operator algebras, that is, the reduced $C^{*}$-algebra $C_{r}^{*}(G)$ and the von Neumann algebra $L(G)$, which are generated by the left regular representation of a discrete group $G$. A discrete group $G$ is called exact if $C_{r}^{*}(G)$ is exact $([10])$, and it was shown by Ozawa [1] that $G$ is exact if and only if $G$ admits an amenable action on a compact space (see [1] for the notion of amenability). Based on this

Received by the editors February 16, 2005 and, in revised form, April 20, 2005.

2000 Mathematics Subject Classification. Primary 46L55; Secondary 46L40, 46L89.

Key words and phrases. Entropy, free group, $C^{*}$-algebra, amenability.

The author was supported in part by JSPS Grant \#14540205.

(C)2006 American Mathematical Society 
fact, Brown-Germain [4 defined the entropy $h a(\alpha)$ for an automorphism $\alpha$ of an exact discrete group.

Let $\alpha$ be an automorphism of a discrete group $G$. Then $\alpha$ induces the automorphism $\hat{\alpha}$ of $C_{r}^{*}(G)$, and $\hat{\alpha}$ is also extended to the automorphism $\overline{\hat{\alpha}}$ of the group von Neumann algebra $L(G)$.

In this paper we restrict our attention to free groups. Let $F_{\infty}$ be the free group with free generators indexed by the integers $\mathbb{Z}$, and assume that $\sigma$ is the automorphism of $F_{\infty}$ which corresponds to the shift $n \rightarrow n+1(n \in \mathbb{Z})$.

As the first typical example of entropy about a highly non-commutative dynamical system, Størmer showed in 12 that $H(\overline{\hat{\sigma}})=0$ for the free shift $\overline{\hat{\sigma}}$ on the type $\mathrm{II}_{1}$ factor $L\left(F_{\infty}\right)$. Later he also proved in [13] that $h_{\phi}(\hat{\sigma})=0$ with respect to the unique $\hat{\sigma}$-invariant state $\phi$ of $C_{r}^{*}\left(F_{\infty}\right)$.

We also see the same phenomenon for the topological entropy in [3] and [8], that is, $h t(\hat{\sigma})=0$.

The purpose of this paper is to show that $h a(\sigma)=0$. This implies all the above results because $h t(\hat{\alpha}) \leq h a(\alpha)$ for all automorphisms $\alpha$ of a group by [4].

The key point of our proof is that the automorphism does not change the length of all reduced words.

\section{Preliminaries}

In this section, we summarize notations, terminologies and basic facts on the entropy $h a(\alpha)$ in 4 for an automorphism $\alpha$ of an exact discrete group $G$.

2.1. Amenable action. Let $G$ be a discrete group and let $\alpha^{G}$ be the canonical action of $G$ on $l^{\infty}(G)$. That is, $\alpha^{G}: G \rightarrow \operatorname{Aut}\left(l^{\infty}(G)\right)$ is a homomorphism given by

$$
\left(\alpha_{g}^{G}(x)\right)(h)=x\left(g^{-1} h\right), \quad \text { for all } \quad x \in l^{\infty}(G), g, h \in G .
$$

Let $l^{1}\left(G, l^{\infty}(G)\right)$ be the closure of the linear space of finitely supported functions $T: G \rightarrow l^{\infty}(G)$ with respect to the norm

$$
\|T\|_{1}=\left\|\sum_{g}|T g|\right\|_{l^{\infty}(G)} .
$$

The action $\alpha^{G}$ is said to be amenable if there exist functions $T_{n} \in l^{1}\left(G, l^{\infty}(G)\right)$ such that $T_{n}$ is non-negative (i.e. $T_{n} g \geq 0$, for all $g \in G$ ), finitely supported, $\sum_{g} T_{n} g=1_{l \infty(G)}$ and $\left\|s \cdot T_{n}-T_{n}\right\|_{1} \rightarrow 0$ for all $s \in G$, where

$$
(s . T) g=\alpha_{s}^{G}\left(T s^{-1} g\right) .
$$

2.2. Entropy $h a(\alpha)$. Assume that the action $\alpha^{G}$ is amenable. Let $\alpha$ be an automorphism of G. By [4, Proposition 2.6], the definition of $h a(\alpha)$ is as follows:

Given a finite subset $\omega \subset G$, and $\delta>0$, let

$$
\operatorname{ra}(\omega, \delta)=\inf \left\{|\operatorname{supp}(T)|:\|s \cdot T-T\|_{1}<\delta, \text { for all } s \in \omega\right\},
$$

where infimum is taken over all finitely supported non-negative functions $T: G \rightarrow$ $l^{\infty}(G)$ which satisfy that $\sum_{g} T g=1$. Here $|\operatorname{supp}(T)|$ denotes the cardinality of the support of $T$. Let

$$
h a(\alpha, \omega, \delta)=\limsup _{n \rightarrow \infty} \frac{\log \left(r a\left(\omega \cup \alpha(\omega) \cup \cdots \alpha^{n-1}(\omega), \delta\right)\right)}{n}
$$


and

$$
h a(\alpha, \omega)=\sup _{\delta>0} h a(\alpha, \omega, \delta) .
$$

Then

$$
h a(\alpha)=\sup _{\omega} h a(\alpha, \omega) .
$$

If $\omega_{1} \subset \omega_{2} \subset \cdots$ are finite sets with the property that $G=\bigcup_{i \in \mathbb{N}} \bigcup_{n \in \mathbb{Z}} \alpha^{n}\left(\omega_{i}\right)$, then $h a(\alpha)=\sup _{i} h a\left(\alpha, \omega_{i}\right)$ by [4, Proposition 5.4].

\section{Results}

Let $I$ be a set, and let $F_{I}$ be the free group with generators which is indexed by the set $I$. Let $\left\{s_{i} ; i \in I\right\}$ be a set of free generators of $F_{I}$, and let $S=\left\{s_{i}, s_{i}^{-1} ; i \in\right.$ $I\}$. Each element $x \in F_{I}$ has a unique expression as a finite product $x=x_{1} x_{2} \cdots x_{k}$ with $x_{i} \in S(i=1,2, \cdots, k)$ and $x_{i+1}^{-1} \neq x_{i}$ for all $i \leq k-1$. The expression is called the reduced word in the letters $S$, and the number $k$ is called the length of the reduced word $x$, which is denoted by $|x|$. We denote by $e$ the empty word with the length 0 , which is the identity of $F_{I}$. For a subset $J \subset I$, we let $S_{J}=\left\{s_{i}, s_{i}^{-1} ; i \in J\right\}$. We denote by $W_{n}(J)$ the set of the reduced words $x$ in the letters $S_{J}$ with $|x|=n$. For a reduced word $x=x_{1} x_{2} \cdots$ (the length does not need to be finite), we let $x_{[i, j]}=x_{i} x_{i+1} \cdots x_{j}$, where $1 \leq i \leq j$.

3.1. Example. Let $J \subset I$ be a finite subset, and let $r$ be a positive integer. We define the map $T_{J, r}: F_{I} \rightarrow l^{\infty}\left(F_{I}\right)$ as follows:

We fix a reduced word with infinite length $w=w_{1} w_{2} \cdots$, which satisfies that

$$
w_{i} \in S_{J} \text { and } w_{i} \notin\left\{w_{1}, w_{2}, \cdots, w_{i-1}\right\}(\bmod 2|J|) \text {, for all } i \text {. }
$$

Case 1. Assume that $z \in F_{I}$ is either the empty word $e$ or the first letter of $z$ is not contained in $S_{J}$, i.e., $z=z_{1} z_{2} \cdots z_{d}$ with $z_{1} \notin S_{J}$. Then we let

$$
Y(J, r ; z)=\left\{w_{[1,2 r]}, w_{[2,2 r]}, \cdots, w_{[r, 2 r]}\right\}
$$

and we let

$$
T_{J, r} y(z)= \begin{cases}r^{-1}, & \text { if } y \in Y(J, r ; z) \\ 0, & \text { otherwise. }\end{cases}
$$

Case 2. Assume that a reduced word $z \in F_{I}$ satisfies that $z=g z^{\prime}$, where $g$ is a reduced word in $S_{J}$ and $z^{\prime}$ is a reduced word such as in Case 1 . We let $g=g_{1} g_{2} \cdots g_{d}$ $\left(g_{i} \in S_{J}\right)$. From the ordered set $\left\{g_{1}, g_{[1,2]}, \cdots, g, g w_{[1,2 r]} g w_{[2,2 r]}, \cdots\right\}$, we choose the set $Y(J, r ; z)$ of the first $r$ different reduced words. That means,

2-1) If $r \leq d$, then

$$
Y(J, r ; z)=\left\{g_{1}, g_{[1,2]}, \cdots, g_{[1, r]}\right\} .
$$

2-2) If $d<r$, then

$$
Y(J, r ; z)=\left\{g_{1}, g_{[1,2]}, \cdots, g, g w_{[1,2 r]}, g w_{[2,2 r]}, \cdots, g w_{[r-d, 2 r]}\right\} .
$$

We let

$$
T_{J, r} y(z)= \begin{cases}r^{-1}, & \text { if } y \in Y(J, r ; z) \\ 0, & \text { otherwise }\end{cases}
$$


3.2. Amenability of $\alpha^{F_{I}}$. The following shows that the $\alpha^{F_{I}}$ is an amenable action of $F_{I}$ on $l^{\infty}\left(F_{I}\right)$.

Lemma. Let $J \subset I$ be a finite subset. Let $x \in F_{J}$, and let $r$ be a positive integer with $|x|<r$. Then the map $T_{J, r}$ is non-negative,

and

$$
\begin{gathered}
\sum_{y \in F_{I}} T_{J, r} y=1_{l^{\infty}\left(F_{I}\right)}, \\
\left|\operatorname{supp}\left(T_{J, r}\right)\right|=|J| \frac{(2|J|-1)^{r}-1}{|J|-1}
\end{gathered}
$$

$$
\left\|T_{J, r}-x \cdot T_{J, r}\right\|_{1} \leq \frac{2|x|}{r} .
$$

Proof. It is obvious that $T_{J, r}$ is non-negative and $\sum_{y \in F_{I}} T_{J, r} y=1$.

Since $\left|W_{n}(J)\right|=2|J|(2|J|-1)^{n-1}$ for all $n \in \mathrm{N}$ and since

$$
\operatorname{supp}\left(T_{J, r}\right)=\bigcup_{n=1}^{r} W_{n}(J)
$$

we have that

$$
\left|\operatorname{supp}\left(T_{J, r}\right)\right|=|J| \frac{(2|J|-1)^{r}-1}{|J|-1} .
$$

To prove that $\left\|T_{J, r}-x \cdot T_{J, r}\right\|_{1} \leq \frac{2|x|}{r}$, we show that $Y(J, r ; z) \cap x Y\left(J, r ; x^{-1} z\right)$ contains at least $(r-|x|)$ reduced words for all $z \in F_{I}$.

Let $m=|x|$, and let $x=x_{1} \cdots x_{m}$, where $x_{i} \in S_{J}$ for all $i$.

Case 1) Assume that $z \in F_{I}$ is such a reduced word as in Case 1 in Example 3.1. Since $m<r$, we have

$$
Y\left(J, r ; x^{-1} z\right)=\left\{x_{m}^{-1}, \cdots, x^{-1}, x^{-1} w_{[1,2 r]}, \cdots, x^{-1} w_{[r-m, 2 r]}\right\}
$$

and

$$
\left\{w_{[1,2 r]}, w_{[2,2 r]}, \cdots, w_{[r-m, 2 r]}\right\} \subseteq Y(J, r ; z) \cap x Y\left(J, r ; x^{-1} z\right) .
$$

Case 2) Assume that $z=g z^{\prime}$, where $g=g_{1} g_{2} \cdots g_{d}\left(g_{i} \in S_{J}\right)$, and $z^{\prime}$ is such a reduced word as in Case 1 in Example 3.1.

2-1) Assume that $r \leq\left|x^{-1} g\right|$.

2-1-1) If $x_{1} \neq g_{1}$, then

$$
Y\left(J, r ; x^{-1} z\right)=\left\{x_{m}^{-1}, \cdots, x^{-1}, x^{-1} g_{1}, \cdots, x^{-1} g_{[1, r-m]}\right\}
$$

and

$$
\left\{g_{1}, g_{[1,2]}, \cdots, g_{[1, r-m]}\right\} \subseteq Y(J, r ; z) \cap x Y\left(J, r ; x^{-1} z\right) .
$$

2-1-2) If there exists an $i$ with $x_{k}=g_{k}$ for all $k(1 \leq k \leq i)$ and $x_{i+1} \neq g_{i+1}$ $\left(x_{i+1}\right.$ may be the identity), then

$$
Y\left(J, r ; x^{-1} z\right)=\left\{x_{m}^{-1}, \cdots, x_{[i+1, m]}^{-1}, x^{-1} g_{[1, i+1]}, \cdots, x^{-1} g_{[1, r-m+2 i]}\right\} \text {. }
$$

2-1-2-1) If $r \leq d$, then

$$
\left\{g_{[1, i+1]}, \cdots, g_{[1, \min (r, r-m+2 i)]}\right\} \subseteq Y(J, r ; z) \cap x Y\left(J, r ; x^{-1} z\right) .
$$

2-1-2-2) If $d<r$, then

$$
\left\{g_{[1, i+1]}, \cdots, g_{[1, r-m+2 i]}\right\} \subseteq Y(J, r ; z) \cap x Y\left(J, r ; x^{-1} z\right) .
$$

2-2) Assume that $\left|x^{-1} g\right|<r$. 
2-2-1) If $x_{1} \neq g_{1}$, then

$$
\begin{aligned}
Y\left(J, r ; x^{-1} z\right)=\left\{x_{m}^{-1}, \cdots, x^{-1}, x^{-1} g_{1},\right. & \cdots, x^{-1} g, \\
& \left.x^{-1} g w_{[1,2 r]}, \cdots, x^{-1} g w_{[r-m-d, 2 r]}\right\}
\end{aligned}
$$

and

$\left\{g_{1}, g_{[1,2]}, \cdots, g, g w_{[1,2 r]}, \cdots, g w_{[r-m-d, 2 r]}\right\} \subseteq Y(J, r ; z) \cap x Y\left(J, r ; x^{-1} z\right)$.

2-2-2) If there exists an $i$ with $x_{k}=g_{k}$ for all $k(1 \leq k \leq i)$ and $x_{i+1} \neq g_{i+1}$ $\left(x_{i+1}\right.$ may be the identity), then

$$
\begin{aligned}
Y\left(J, r ; x^{-1} z\right)=\left\{x_{m}^{-1}, \cdots, x_{[i+1, m]}^{-1},\right. & x^{-1} g_{[1, i+1]}, \cdots, x^{-1} g, \\
& \left.x^{-1} g w_{[1,2 r]}, \cdots, x^{-1} g w_{[r-m-d+2 i, 2 r]}\right\} .
\end{aligned}
$$

2-2-2-1) If $r \leq d$, then

$$
\left\{g_{[1, i+1]}, \cdots, g_{[1, r]}\right\} \subseteq Y(J, r ; z) \cap x Y\left(J, r ; x^{-1} z\right) .
$$

2-2-2-2) If $d<r$, then

$$
\begin{aligned}
\left\{g_{[1, i+1]}, \cdots, g, g w_{[1,2 r]}, \cdots, g w_{[\min (r-d, r-m-d+2 i), 2 r]}\right\} & \\
& \subseteq Y(J, r ; z) \cap x Y\left(J, r ; x^{-1} z\right) .
\end{aligned}
$$

Thus $Y(J, r ; z) \cap x Y\left(J, r ; x^{-1} z\right)$ always contains at least $(r-|x|)$ reduced words for all $z \in F_{I}$.

Denote the symmetric difference of two sets $Y_{1}$ and $Y_{2}$ by $Y_{1} \nabla Y_{2}$. Since

$$
\left\{y \in F_{I}: T_{J, r} x^{-1} y\left(x^{-1} z\right)-T_{J, r} y(z) \neq 0\right\} \subseteq x Y\left(J, r ; x^{-1} z\right) \nabla Y(J, r ; z),
$$

we have by the definition of $T_{J, r}$

$$
\begin{aligned}
\sum_{y \in F_{I}} & \left|T_{J, r} x^{-1} y\left(x^{-1} z\right)-T_{J, r} y(z)\right| \\
\leq & \frac{\left|x Y\left(J, r ; x^{-1} z\right) \nabla Y(J, r ; z)\right|}{r} \\
& \leq \frac{2|x|}{r} .
\end{aligned}
$$

Hence

$$
\left\|T_{J, r}-x \cdot T_{J, r}\right\|_{1}=\sup _{z \in F_{I}} \sum_{y \in F_{I}}\left|T_{J, r} y(z)-T_{J, r} x^{-1} y\left(x^{-1} z\right)\right| \leq \frac{2|x|}{r} .
$$

3.3. Entropy of automorphisms by permutations. The following theorem gives as the special case that the free shift automorphism of the free group $F_{\infty}$ has zero entropy.

Theorem. Let $\sigma \in A u t\left(F_{I}\right)$ be the automorphism arising from a permutation $\sigma$ of the index set $I$. Then $h a(\sigma)=0$.

Proof. Given a finite subset $J \subset I$ and a positive integer $m$, we let

$$
\omega(J, m)=\bigcup_{i=0}^{m} W_{i}(J) .
$$

Then $\bigcup_{J \subset I} \bigcup_{m=0}^{\infty} \omega(J, m)=F_{I}$. 
Hence it is sufficient to show, for all finite subsets $J \subset I$ and $m \in \mathbb{N}$, that $h a(\sigma, \omega(J, m), \delta)=0$ for any $\delta>0$.

Let $\Im(J, n)=\bigcup_{i=0}^{n-1} \sigma^{i}(J)$. Then

$$
\bigcup_{i=0}^{n-1} \sigma^{i}(\omega(J, m)) \subseteq \omega(\Im(J, n), m) .
$$

Given $\delta>0$, we choose $r \in \mathbb{N}$ with $2 m<\delta r$.

Then by the Lemma, $T_{\Im(J, n), r}$ satisfies that

$$
\left\|T_{\Im(J, n), r}-x \cdot T_{\Im(J, n), r}\right\|_{1} \leq \frac{2|x|}{r} \leq \frac{2 m}{r}<\delta, \quad \text { for all } \quad x \in \omega(\Im(J, n), m) .
$$

Since $|\Im(J, n)| \leq n|J|$, it follows again by the Lemma that

$$
r a\left(\bigcup_{i=0}^{n-1} \sigma^{i}(\omega(J, m)), \delta\right) \leq\left|\operatorname{supp}\left(T_{\Im(J, n), r}\right)\right| \leq n|J| \frac{(2 n|J|-1)^{r}-1}{n|J|-1} .
$$

Hence

$$
h a(\sigma, \omega(J, m), \delta) \leq \limsup _{n \rightarrow \infty} \frac{1}{n} \log \left(n|J| \frac{(2 n|J|-1)^{r}-1}{n|J|-1}\right)=0 .
$$

3.4. Applications to entropy in the operator algebras. As applications of the above theorem to the theory of operator algebras, we have the following:

Corollary. Let $\sigma \in \operatorname{Aut}\left(F_{I}\right)$ be the automorphism arising from a permutation $\sigma$ of the index set $I$. Then

$$
h a(\sigma)=h t(\hat{\sigma})=h t_{\phi}(\hat{\sigma})=h_{\phi}(\hat{\sigma})=H(\overline{\hat{\sigma}})=0,
$$

where $\phi$ is a $\hat{\sigma}$ invariant state of $C_{r}^{*}\left(F_{I}\right)$.

Proof. Let $\alpha$ be an automorphism of an exact discrete group $G$. It always holds that $h a(\alpha) \geq h t(\hat{\alpha})$ by [4, Proposition 3.3]. If $\beta$ is an automorphism of an exact $C^{*}$-algebra, then $h t(\beta) \geq h_{\phi}(\beta)$ by [8. Proposition 9 ], and also for the modified dynamical entropy $h t_{\phi}(\beta)$ of $h t(\beta)$, we have that $h t_{\phi}(\beta) \leq h t(\beta)$ by [5] (cf. [9]). Furthermore, if $\phi$ is a tracial state, then $h_{\phi}(\beta)=H(\bar{\beta})$ by [7].

The author thanks the referee for kind comments and advice in writing the proof of Lemma 3.2 in detail.

\section{REFERENCES}

1. C. Anantharaman-Delaroche and J. Renault, Amenable groupoids, Monographs of L'Enseignement Mathematique, 36 Geneva, 2000. MR1799683 (2001m:22005)

2. N. Brown, Topological entropy in exact $C^{*}$-algebras, Math. Ann., 314 (1999), 347-367. MR 1697449 (2000g:46093)

3. N. Brown and M. Choda, Approximation entropies in crossed products with an application to free shifts, Pacific J. Math., 198 (2001), 331-346. MR.1835512 (2002b:46105)

4. N. Brown and E. Germain, Dual entropy in discrete groups with amenable actions, Erg. Th. and Dynam. Sys., 22 (2002), 711-728. MR1908551 (2003c:37021)

5. M. Choda, Dynamical entropy for automorphisms of exact $C^{*}$-algebras, Jour. Funct. Anal., 198 (2003), 481-493. MR1964548(2004b:46093)

6. A. Connes and E. Störmer, Entropy of II 1 von Neumann algebras, Acta Math., 134 (1975), 289-306. MR0454657 (56:12906) 
7. A. Connes, H. Narnhofer and W. Thirring, Dynamical entropy of $C^{*}$ algebras and von Neumann algebras, Commun. Math. Phys., 112 (1987), 691-719. MR0910587 (89b:46078)

8. K. Dykema, Topological entropy of some automorphisms of reduced amalgamated free product $C^{*}$-algebras, Ergod. Th. \& Dynam. Sys., 21 (2001), 1683-1693. MR1869065 (2002i:46062)

9. D. Kerr and C. Pinzari, Noncommutative pressure and the variational principle in CuntzKrieger-type $C^{*}$-algebras, Jour. Funct. Anal., 188 (2002), 156-215. MR.1878635(2003f:46110)

10. E. Kirchberg and S. Wassermann, Exact groups and continuous bundles of $C^{*}$-algebras, Math. Ann., 315 (1999), 169-203. MR.1721796 (2000i:46050)

11. N. Ozawa, Amenable actions and exactness for discrete groups, C. R. Acad. Sci. Paris Ser. I Math., 330 (2000), 691-695. MR1763912 (2001g:22007)

12. E. Störmer, Entropy of some automorphisms of the $I I_{1}$ factor of the free group in infinite number of generators, Invent. Math. 110 (1992), 63-73. MR1181816 (93i:46112)

13. E. Störmer, States and shifts on infinite free products of $C^{*}$-algebras, Fields Inst. Comm., 12 (1997), 281-291. MR 1426846 (98d:46077)

14. E. Störmer, A survey of noncommutative dynamical entropy. Classification of nuclear $C^{*}$ algebras. Entropy in operator algebras, 147-198, Encyclopaedia Math. Sci., 126, Springer, Berlin, 2002. MR $1878883(2002 \mathrm{k}: 46175)$

15. D. Voiculescu, Dynamical approximation entropies and topological entropy in operator algebras, Commun. Math. Phys., 170 (1995), 249-281. MR1334396 (97b:46082)

Department of Mathematics, Osaka Kyolku University, Asahigaoka, Kashiwara 582 8582, JAPAN

E-mail address: marie@cc.osaka-kyoiku.ac.jp 